\title{
Histoplasma capsulatum in peripheral blood neutrophils
}

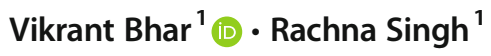

Received: 6 August 2019 / Accepted: 4 February 2020 /Published online: 2 March 2020

(C) Springer-Verlag GmbH Germany, part of Springer Nature 2020

\section{Case report}

A 45-year-old female presented with chief complaints of moderate- to high-grade fever for 4 months associated with drenching night sweats. Physical examination revealed hepatosplenomegaly, 10 and $12 \mathrm{~cm}$ below costal margins, respectively. Her complete blood count showed anemia $(78 \mathrm{~g} / \mathrm{L})$, thrombocytopenia $\left(20 \times 10^{9} / \mathrm{L}\right)$, and total leukocyte count $\left(4.6 \times 10^{9} / \mathrm{L}\right)$. The differential leukocyte count (DLC) at presentation was as follows: neutrophils $80 \%$, lymphocytes $13 \%$, eosinophils $04 \%$, monocytes $01 \%$, myelocytes $01 \%$, and metamyelocytes $01 \%$. The patient had a history of seronegative rheumatoid arthritis and was taking methotrexate or azathioprine intermittently. A peripheral blood smear obtained on admission showed a few neutrophils with one to multiple, small, intracellular yeast-like organisms with crescent-like eccentric chromatin. The organisms were ovoid in shape and surrounded by a cytoplasmic halo (Fig. 1a; original magnification $\times 1000$, Leishman stain). Periodic acid-Schiff (PAS) stain highlighted the rim of these organisms. These features are consistent with diagnosis of Histoplasma capsulatum var. capsulatum. Subsequent bone marrow aspiration and biopsy were performed which revealed numerous histiocytes filled with $H$. capsulatum yeast forms. Some of the histiocytes show hemophagocytosis (Fig. $1 \mathrm{~b}$ and c; original magnification $\times$ 1000, May-Grünwald Giemsa and Gomori-methenamine silver stain). The patient was treated with amphotericin $\mathrm{B}$ and showed remarkable clinical improvement.

$H$. capsulatum is acquired by the inhalation of conidia or mycelial fragments [1]. Disseminated histoplasmosis is

Vikrant Bhar

vikrantbhar86@gmail.com

1 Artemis Hospital, Gurugram, India a severe and often fatal opportunistic infection, when left untreated, among patients with immunodeficiency that includes patients with acquired immunodeficiency syndrome (AIDS), those with hematologic malignancies, and those on immunosuppressive therapy. Our patient was on treatment with azathioprine intermittently for her medical condition. There are various tests which can be used to diagnose $H$. capsulatum infection, including mycology cultures, histopathological examination, polymerase chain reaction-based methods, and serological tests. The morphological identification of distinctive 2- to 4- $\mu \mathrm{m}$, oval, narrow base budding yeast forms with surrounding halo (clear zone) corresponding to the cell wall allows a tentative diagnosis of $H$. capsulatum var. capsulatum infection. The cell wall is highlighted with PAS and GMS stains. In addition, clustering of yeast forms within macrophages is an important diagnostic feature of Histoplasma infection. Leishmania and Candida glabrata can mimic $H$. capsulatum var. capsulatum morphologically; however, Leishmania amastigotes are oval bodies, mostly present intracellularly in macrophages and macrocytes, 2-3 $\mu \mathrm{m}$ in length, have rod-like body called kinetoplast oriented right angle to the nucleus, and absence of peripheral halo as noted in Histoplasma. Candida glabrata does not cause a clinical syndrome similar to histoplasmosis, usually results in suppurative inflammatory response, and depict size heterogeneity compared with $H$. capsulatum var. capsulatum and mostly extracellular distribution of organisms $[1,2]$.

The peripheral blood histoplasmosis is uncommon and a few case reports have been published $[3,4]$. The secondary hemophagocytosis is a well-known complication of disseminated $H$. capsulatum infection [5].

This case highlights the significance of careful examination of peripheral blood smear to pick up clinically useful rare findings. 
Fig. 1 a Neutrophil with multiple yeast-like organisms with crescent like eccentric chromatin (1000×, Leishman stain). b, c Histiocytes filled with $H$. capsulatum yeast forms (original magnification 1000x, MayGrunwald Giemsa and GomoriMethenamine silver stain)

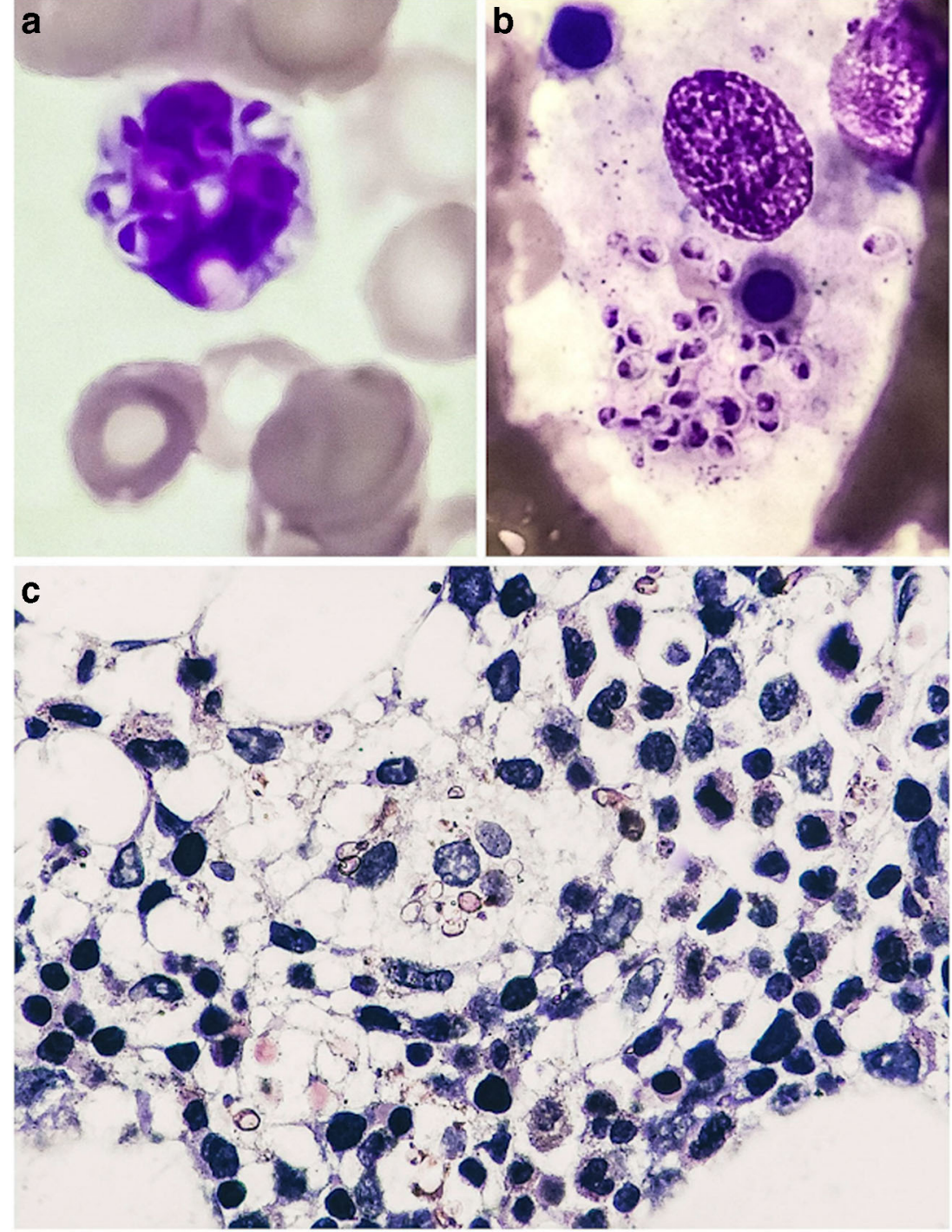

\section{Compliance with ethical standards}

Conflict of interest The authors declare that they have no conflict of interest.

\section{References}

1. Kauffman CA (2007) Histoplasmosis: a clinical and laboratory update. Clin Microbiol Rev 20(1):115-132

2. Guarner J, Brandt ME (2011) Histopathologic diagnosis of fungal infections in the 21st century. Clin Microbiol Rev 24(2):247-280
3. Updesh M, Sachdeva S, Das R (2010) Peripheral smear discloses histoplasmosis. Blood. 115(18):3653

4. Maltos AL, Maia E, Oliveira CD (2017) Disseminated histoplasmosis diagnosed in a peripheral blood smear. Rev Soc Bras Med Trop 50(1):148

5. Townsend JL, Shanbhag S, Hancock J, Bowman K, Nijhawan AE (2015) Histoplasmosis-induced hemophagocytic syndrome: a case series and review of the literature. Open Forum Infect Dis 2(2): ofv055

Publisher's note Springer Nature remains neutral with regard to jurisdictional claims in published maps and institutional affiliations. 